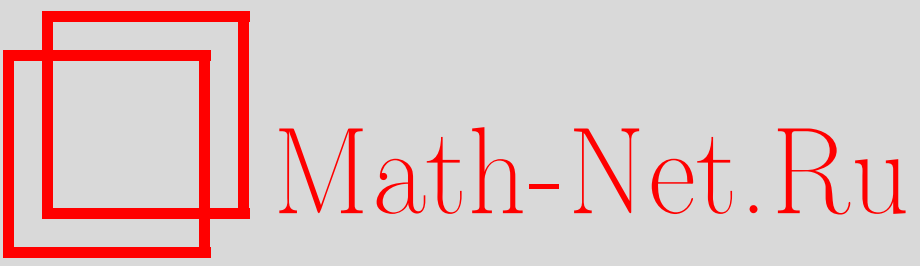

Ю. С. Вернов, М. Н. Мнацаканова, Коммутационные соотношения в пространстве с индефинитной метрикой, ТМФ, 2003, том 135, номер 3, 420-426

DOI: https://doi.org/10.4213/tmf204

Использование Общероссийского математического портала Math-Net.Ru подразумевает, что вы прочитали и согласны с пользовательским соглашением

http://www.mathnet.ru/rus/agreement

Параметры загрузки:

IP: 18.207 .199 .55

26 апреля 2023 г., 13:24:52 
ТЕОРЕТИЧЕСКАЯ

И МАТЕМАТИЧЕСКАЯ

ФИЗИКА

Tом 135, № 3

июнь, 2003

(C) 2003 г. $\quad$ Ю. С. Вернов* ${ }^{*}$ М.Н. Мнацаканова

\section{КОММУТАЦИОННЫЕ СООТНОШЕНИЯ В ПРОСТРАНСТВЕ С ИНДЕФИНИТНОЙ МЕТРИКОЙ}

Дано описание неприводимых регулярных представлений алгебры операторов $a$ и $b$, определяемой соотношениями $[a, b]=1, b a=a^{+} b^{+}$, в произвольном невырожденном, замкнутом пространстве с индефинитной метрикой. Найдена связь этой алгебры с обобщенной алгеброй Гейзенберга.

Ключевые слова: индефинитная метрика, алгебра Гейзенберга, регулярные представления.

Алгебра Гейзенберга, т.е. алгебра канонических коммутационных соотношений

$$
\left[a, a^{+}\right]=1
$$

лежит в основе квантовой теории поля. Поэтому изучению свойств этой алгебры посвяшено большое число исследований начиная с классических работ Гейзенберга и Шредингера (обзор основных результатов можно найти в [1]). Поскольку теория калибровочных полей в ковариантной формулировке требует использования пространства с индефинитной метрикой [2]-[4], наиболее важньй класс представлений алгебры Гейзенберга, названный регулярным, был изучен в таком пространстве [5], [6]. Условие регулярности, справедливое как для самой алгебры Гейзенберга, так и для ее обобщений, сформулировано ниже (см. определение). В последние годы широко изучались $q$ - и $R$-деформированные алгебры Гейзенберга (см. соответственно работы [7], [8] и [9], [10] и ссылки в них). Свойства регулярных представлений этих алгебр в пространстве с индефинитной метрикой изучены в работах [11]-[13]. Указанные алгебры являются частными случаями более общей алгебры, определяемой соотношениями

$$
\begin{gathered}
a^{+} a=\varphi(N), \quad a a^{+}=\varphi(N+1), \\
N=N^{+}, \quad[N, a]=-a,
\end{gathered}
$$

где $\varphi(N)$ - некоторая функция. Отметим, что соотношение (1) или его обобшения, в частности $q$ - и $R$-деформации, не фиксируют однозначно связь между $a^{+} a\left(a a^{+}\right)$и оператором $N$, определенным условиями (3). Действительно, например, в случае алгебры

\footnotetext{
${ }^{*}$ Институт ядерных исследований РАН, Москва, Россия. E-mail: vernov@ms2.inr.ac.ru

${ }^{\dagger}$ Научно-исследовательский институт ядерной физики им. Д.В. Скобельцына, Московский государственный университет, Москва, Россия. E-mail: mnatsak@theory.sinp.msu.ru
} 
Гейзенберга оператор $N \equiv a^{+} a+C \mathbb{I}$, где $\mathbb{I}$ - единичный оператор, удовлетворяет условиям (3) при любом $C \in \mathbb{R}$. В случае $q$-деформированной алгебры Гейзенберга эта неоднозначность носит нетривиальный характер:

$$
a^{+} a=\frac{C q^{N}-q^{-N}}{q-q^{-1}}, \quad C \in \mathbb{R}
$$

(см. [12], формулы (26), $\left.\left(26^{\prime}\right)\right)$. Однако при любом фиксированном $C$ изучение регулярных представлений этой алгебры сводится к исследованию соответствующей обобщенной алгебры Гейзенберга.

Такая алгебра была введена в работе [6]. В [12], [13] ее свойства рассматривались в связи с изучением $q$ - и $R$-деформаций алгебры Гейзенберга. В [14] были изучены свойства этой алгебры, не зависящие от конкретного вида функции $\varphi(N)$. Например, было показано, что ее произвольное регулярное представление реализуется в пространстве Крейна, базисом которого являются собственные векторы оператора $N$. Свойства этих векторов зависят от $\varphi(N)$, и, более того, при одной и той же функции $\varphi(N)$ существуют представления в различных пространствах Крейна (см., например, [5]).

Мы рассмотрим другое обобщение алгебры Гейзенберга, а именно алгебру $A$, определяемую соотношениями

$$
\begin{gathered}
{[a, b]=1,} \\
N=N^{+}, \quad N=b a .
\end{gathered}
$$

Коммутационные свойства операторов исследовались в пространстве Гильберта [1]. Представляет интерес изучение свойств алгебры, заданной соотношениями (4) и (5), в более общем пространстве.

Мы изучим регулярные представления этой алгебры в произвольном невырожденном и замкнутом (в сильном или слабом смысле) пространстве $J$, в котором задано, вообще говоря, индефинитное скалярное произведение $\langle\cdot, \cdot\rangle$. Напомним, что пространство $J$ невырожденно, если из условия $x \perp J$ следует, что $x=0$. Будет доказано, что, как и в случае алгебры Гейзенберга, спектр оператора $N$ может быть трех типов: $\operatorname{Sp} N=\mathbb{N}$; $\operatorname{Sp} N=\mathbb{Z}^{-} ; \operatorname{Sp} N=\lambda+\mathbb{Z}, \lambda \in \mathbb{R}$. Однако, в отличие от алгебры Гейзенберга, где спектр оператора $N$ определяет представление с точностью до унитарной эквивалентности, в рассматриваемой алгебре при одном и том же спектре $N$ сушествует множество унитарно-неэквивалентных представлений. Это связано с тем, что представление определяется не только операторами $a$ и $b$, но и их сопряженными.

В работе доказано, что каждое неприводимое представление указанной алгебры унитарно-эквивалентно некоторому неприводимому представлению обобшенной алгебры Гейзенберга (см. ниже (17), (22)). Доказано, что, в свою очередь, обобшенная алгебра Гейзенберга может быть преобразована в алгебру, заданную соотношениями (4), (5), за исключением того случая, ког да соответствующий оператор $N$ имеет конечный спектр.

Показано, что рассматриваемая алгебра может быть преобразована в алгебру Гейзенберга, если метрики пространств соответствующих представлений совпадают (см. ниже (25), (26)). 
ОПредЕлЕниЕ. Представление алгебры $A$ называется регулярным, если существует оператор $U(s)$, удовлетворяющий следуюшим условиям:

$$
\begin{gathered}
U(s) U(t)=U(s+t), \\
U(s) \rightarrow \mathbb{I} \text { при } s \rightarrow 0, \quad U(0)=\mathbb{I}, \\
\lim _{s \rightarrow 0} \frac{U(s)-\mathbb{I}}{s} \psi=i N \psi, \text { если } \psi \in D(N),
\end{gathered}
$$

II - единичный оператор.

ЗАмЕчАниЕ 1. В случае алгебры Гейзенберга для представлений в гильбертовом пространстве это определение эквивалентно условиям Реллиха-Диксмье [15]. Для таких представлений канонические коммутационные соотношения могут быть записаны как в обычной $(1)$, так и в вейлевской форме (см. $[1], \S 4)$.

ЗАмЕчАниЕ 2. Представление будет регулярным и в том случае, если условия (6) выполнены только в некоторой области $D$, инвариантной относительно $U(s)$, однако оказывается, что в действительности $U(s)$ - ограниченный оператор и, следовательно, определен во всем пространстве $J$.

Отметим, что сами операторы $a$ и $b$ не ограничены (см. [1], с. 2). Поэтому соотношение (4) выполнено не во всем пространстве $J$, а лиш в $D(N)$. Из самосопряженности $N$ следует, что оператор $U(s)$ унитарен, причем $U^{+}(s)=U(-s)$.

Спектр оператора $N$ найдем с помощью рассуждений, аналогичных проведенным в работе [5] для алгебры Гейзенберга. Сначала заметим, что согласно соотношению (4) получаем

$$
[N, a]=-a, \quad[N, b]=b .
$$

Эти соотношения, очевидно, эквивалентны равенствам

$$
N a=a(N-1), \quad N b=b(N+1) .
$$

Из условий (7) легко устанавливаются правила коммутации оператора $U(s)$ с $a$ и $b$, а именно

$$
U(s) a=e^{-i s} a U(s), \quad U(s) b=e^{i s} b U(s) .
$$

Соотношения (8) следуют из равенств

$$
\begin{aligned}
\frac{d}{d s} U(s) a U(-s) & =i U(s)[N, a] U(-s)=-i U(s) a U(-s), \\
\frac{d}{d s} U(s) b U(-s) & =i U(s)[N, b] U(-s)=i U(s) b U(-s)
\end{aligned}
$$

которые, в свою очередь, суть следствия формул (7) и равенства

$$
\frac{d}{d s} U(s)=i N U(s)
$$

непосредственно вытекаюшего из условий (6). 
Согласно (8)

$$
[U(2 \pi), a]=[U(2 \pi), b]=\left[U(2 \pi), a^{+}\right]=\left[U(2 \pi), b^{+}\right]=0
$$

Два последних равенства следуют из соотношений

$$
[U(-2 \pi), a]=[U(-2 \pi), b]=0 .
$$

Для неприводимого представления условия (11) означают, что

$$
U(2 \pi)=e^{2 \pi i \lambda}, \quad \lambda \in \mathbb{R}
$$

(см. обсуждение подобного вопроса в работе [5]). Из условия (12) следует, что оператор $N$ имеет собственный вектор:

$$
N \psi_{\lambda}=\lambda \psi_{\lambda}
$$

Доказательство этого утверждения аналогично проведенному в [5] для алгебры Гейзенберга, для обобшенной алгебры Гейзенберга оно приведено в [14].

Из условий (7) следует, что спектр оператора $N$ состоит из чисел $\lambda+k, k \in \mathbb{Z}$, а все собственные векторы оператора $N$, принадлежашие неприводимому представлению, имеют вид

$$
\psi_{\lambda+n}=b^{n} \psi_{\lambda}, \quad \psi_{\lambda-n}=a^{n} \psi_{\lambda}, \quad n \in \mathbb{Z}^{+} .
$$

Далее легко видеть, что

$$
M\left(a^{n}, b^{m}\right) \psi_{\lambda} \sim \psi_{\lambda+m-n}
$$

где $M\left(a^{n}, b^{m}\right)$ - произвольный моном от $n$ операторов $a$ и $m$ операторов $b$. Следовательно,

$$
P_{n}(a, b) \psi_{\lambda}=\sum_{k} C_{k} \psi_{\lambda+k}
$$

где $P_{n}(a, b)$ - произвольный полином от операторов $a$ и $b$. Таким образом, действие полиномов от операторов $a$ и $b$ на $\psi_{\lambda}$ порождает пространство $J_{0}-$ пространство конечных линейных комбинаций векторов $\psi_{\lambda+k}$. Из формулы (15) непосредственно следует, что любой собственный вектор оператора $N$, принадлежащий рассматриваемому неприводимому представлению, как и любой вектор $x \in J_{0}$, является циклическим для этого представления. Таким образом, построенное представление является неприводимым, а $J=\bar{J}_{0}$, где $\bar{J}_{0}$ - замыкание пространства $J_{0}$. Явное построение $\bar{J}_{0}$ аналогично соответствуюшей процедуре для обобщенной алгебры Гейзенберга [14].

Найдем теперь типы спектров оператора $N$. Покажем, что, как и для алгебры Гейзенберга, возможны три случая: $\operatorname{Sp} N=\mathbb{N} ; \operatorname{Sp} N=\mathbb{Z}^{-} ; \operatorname{Sp} N=\lambda+\mathbb{Z}, \lambda \in \mathbb{R}$.

Действительно, пусть $a \psi_{\lambda-n}=0$. Это означает, что $b a \psi_{\lambda-n}=(\lambda-n) \psi_{\lambda-n}=0$, т.е. $\lambda=n$. В этом случае все $b^{n} \psi_{\lambda} \neq 0$. Действительно, если $b^{k} \psi_{\lambda} \neq 0, k=0,1, \ldots, n-1$, а $b^{n} \psi_{\lambda}=0$, то $a b b^{n-1} \psi_{\lambda}=2 n b^{n-1} \psi_{\lambda}=0$, что противоречит предположению, что $b^{n-1} \psi_{\lambda} \neq 0$. Пусть теперь $b^{n} \psi_{\lambda}=0$, тогда $\lambda=-n$, и рассуждая аналогично, убеждаемся, что в этом случае спектр $N$ не ограничен снизу. Отметим, что возможность того, 
что $b a \psi_{\lambda-n}=0, a \psi_{\lambda-n} \neq 0$ или $a b \psi_{\lambda+n-1}=0, b \psi_{\lambda+n-1} \neq 0$, исключена, так как в этих случаях вектор $\psi_{\lambda}$ не принадлежит рассматриваемому представлению. Из проведенного рассмотрения следует, что если $\lambda \notin \mathbb{Z}$, то все $\lambda+k, k \in \mathbb{Z}$, принадлежат $\operatorname{Sp} N$. Итак, как и в случае алгебры Гейзенберга [5], регулярные неприводимые представления разбиваются на три класса:

1) $\operatorname{Sp} N=\mathbb{N}$, в качестве циклического вектора естественно взять $\psi_{0}$;

2) $\operatorname{Sp} N=\mathbb{Z}^{-}$, в качестве циклического вектора выбираем $\psi_{-1}$;

3) $\operatorname{Sp} N=\lambda+\mathbb{Z}$, в качестве циклического вектора всегда можно взять вектор $\psi_{\lambda}$, $-1<\lambda<0$.

В случае алгебры Гейзенберга сушествует однозначная связь между метрикой пространства $J$ и классом представления [5]. А именно, для представления первого класса метрика дефинитная (мы всегда можем выбрать ее положительной), а для двух других классов - индефинитная, причем выбор знака $\left\langle\psi_{\lambda}, \psi_{\lambda}\right\rangle$ однозначно определяет знак $\left\langle\psi_{\lambda+k}, \psi_{\lambda+k}\right\rangle$.

Для рассматриваемой алгебры метрика пространства, соответствуюшего определенному неприводимому представлению, определяется операторами $a^{+}, b^{+}$, и мы имеем широкий спектр возможностей.

Из условий (7) и самосопряженности оператора $N$ следует, что

$$
\left[N, a^{+}\right]=a^{+}, \quad\left[N, b^{+}\right]=-b^{+}
$$

Поэтому $a^{+} \psi_{\lambda+k} \sim \psi_{\lambda+k+1}, b^{+} \psi_{\lambda+k} \sim \psi_{\lambda+k-1}$, т.е.

$$
a^{+}=\xi(N) b, \quad a=b^{+} \xi(N)
$$

Из полученных ниже формул $(23),(24)$ следует, что $\xi(N)=\xi^{+}(N)$. Согласно (17) в качестве базисных векторов можно вместо $b^{n} \psi_{\lambda}$ взять $\left(a^{+}\right)^{n} \psi_{\lambda}$. Легко видеть, что справедлива следующая рекуррентная формула:

$$
\left\langle\left(a^{+}\right)^{n} \psi_{\lambda},\left(a^{+}\right)^{n} \psi_{\lambda}\right\rangle=\xi(\lambda+n)(\lambda+n)\left\langle\left(a^{+}\right)^{n-1} \psi_{\lambda},\left(a^{+}\right)^{n-1} \psi_{\lambda}\right\rangle
$$

Эта формула - непосредственное следствие равенств (17) и того, что согласно (5) $b^{+} a^{+}=$ $N+1$. Кроме того, из условий $\left(7^{\prime}\right)$ следует

$$
\xi(N) a=a \xi(N-1), \quad \xi(N) b=b \xi(N+1) .
$$

Полагая $a a^{+}\left(a^{+}\right)^{n-1} \psi_{\lambda}=\varphi(\lambda+n)\left(a^{+}\right)^{n-1} \psi_{\lambda}$, видим, что

$$
\varphi(\lambda+n)=\xi(\lambda+n)(\lambda+n)
$$

Из формул (18) и (19) получаем

$$
\left\langle\left(a^{+}\right)^{n} \psi_{\lambda},\left(a^{+}\right)^{n} \psi_{\lambda}\right\rangle=\varphi(\lambda+n) \ldots \varphi(\lambda+1)\left\langle\psi_{\lambda}, \psi_{\lambda}\right\rangle
$$


Аналогичным образом приходим к равенству

$$
\left\langle a^{n} \psi_{\lambda}, a^{n} \psi_{\lambda}\right\rangle=\varphi(\lambda-n+1) \ldots \varphi(\lambda)\left\langle\psi_{\lambda}, \psi_{\lambda}\right\rangle
$$

где

$$
\varphi(\lambda-m)=\xi(\lambda-m)(\lambda-m) .
$$

(Формулы (20), (21) совпадают с формулами (15), (16) в работе [14].) Согласно (19) и $(22)$

$$
a a^{+}=\varphi(N+1)=\xi(N+1)(N+1) .
$$

Далее, рассматривая оператор $a^{+} a a^{+}$, легко убедиться в том, что

$$
a^{+} a=\varphi(N) \text {. }
$$

Поскольку оператор $\varphi(N)$ самосопряжен, то из формул $(23),(24)$ следует самосопряженность $\xi(N)$. Сравнивая формулы $(23),(24)$ с равенствами (2), видим, что операторы $a$ и $a^{+}$образуют обобщенную алгебру Гейзенберга. Следовательно, рассматриваемая алгебра операторов $a$ и $b$ при заданной функции $\xi(N)$ изоморфна некоторой обобщенной алгебре Гейзенберга.

Можно сказать, что рассмотренная алгебра является "промежуточной" между самой алгеброй Гейзенберга и обобщенной алгеброй Гейзенберга. Спектр оператора $N$ совпадает со спектром аналогичного оператора в алгебре Гейзенберга, но метрика может быть произвольной. В свою очередь, любая обобшенная алгебра Гейзенберга с помошью преобразования (17) может быть сведена к алгебре, определяемой соотношениями (4) и (5), разумеется, при указанных выше ограничениях на спектр оператора $N$. Отметим, что поскольку для любой обобшенной алгебры Гейзенберга (2) оператор $\widetilde{N}=N+C \mathbb{I}, C \in \mathbb{R}$, также удовлетворяет условиям (3), то ее можно представить в виде $a^{+} a=\tilde{\varphi}(\tilde{N}), a a^{+}=\tilde{\varphi}(\tilde{N}+1)$. При соответствующем выборе $C$ записанная в таком виде обобщенная алгебра Гейзенберга может быть связана с рассматриваемой в работе алгеброй соотношениями (17). Исключением являются представления, в которых спектр оператора $N$ конечен.

В заключение покажем, что если не только спектр, но и метрика представления рассмотренной алгебры те же, что и в соответствуюшем представлении алгебры Гейзенберга: $\left[c, c^{+}\right]=1$, то эти две алгебры связывает простое преобразование. А именно,

$$
\begin{aligned}
c & =a \mu(N), \\
c^{+} & =\nu(N) b .
\end{aligned}
$$

Из условия $c^{+} c=N$ следует, что $\nu(N)=\mu^{-1}(N)$. Далее из равенств $(25),(17)$ и (19) получаем

$$
\mu^{+}(N) \mu(N)=\xi^{-1}(N) .
$$

Согласно (19) и (22) в любом неприводимом представлении (см. также [5], § 3) $\xi(\lambda+k)>0 \forall k, k \in \mathbb{Z}$. Запишем $\mu(N)$ и $\mu^{+}(N)$ в виде

$$
\mu(N)=\tilde{\mu}(N) V(N), \quad \mu^{+}(N)=V^{+}(N) \tilde{\mu}(N),
$$


где $V(N)$ - некоторый унитарный оператор. Согласно (27)

$$
\tilde{\mu}(\lambda+k)=\xi^{-1 / 2}(\lambda+k), \quad k \in \mathbb{Z} .
$$

Легко видеть, что операторы $c^{\prime}=c V(N), c^{++}=V^{+}(N) c^{+}$так же, как и $c, c^{+}$, удовлетворяют алгебре Гейзенберга.

\section{Список литературы}

[1] C.R. Putnam. Commutation Properties of Hilbert Space Operators and Related Topics. Berlin-Heidelberg-New York: Springer, 1967.

[2] F. Strocchi, A.S. Wightman. J. Math. Phys. 1974. V. 15. P. 2198.

[3] G. Morchio, F. Strocchi. Ann. Inst. H. Poincaré. 1980. V. 33. P. 251.

[4] F. Strocchi. Selected Topics on the General Properties of Quantum Field Theory. Singapore: World Scientific, 1993.

[5] M. Mnatsakanova, G. Morchio, F. Strocchi, Yu. Vernov. J. Math. Phys. 1998. V. 29. P. 2969.

[6] M.N. Mnatsakanova, G. Morchio, Yu.S. Vernov. Canonical commutation relations in a positive and an indefinite metric space. In: Quarks-96. Proc. 9th Int. Seminar (Yaroslavl, Russia, May 5-11, 1996). V. 2. Eds. V.A. Matveev, A. A. Penin, V. A. Rubakov, A. N. Tavkhelidze. M.: Inst. Nucl. Res., Russ. Acad. Sci., 1997. P. 51.

[7] M. Chaichian, P. P. Kulish. Phys. Lett. B. 1990. V. 234. P. 72.

[8] P. P. Kulish, E. V. Damaskinsky. J. Phys. A. 1990. V. 23. P. L415; P. P. Kulish. TM . 1991. T. 86. C. 8.

[9] M.S. Plyushchay. Nucl. Phys. B. 1997. V. 491. P. 619.

[10] M.S. Plyushchay. Mod. Phys. Lett. A. 1996. V. 11. P. 2953.

[11] M. Chaichian, M. N. Mnatsakanova, Yu.S. Vernov. Analogue of von Neumann's theorem of the $q$-oscillator algebra. In: Proc. of the 15th Workshop "Problems on High Energy Physics and Field Theory" (Protvino, 1992). Eds. A. P. Samokhin, G. L. Rcheulishvili. Protvino: Inst. High. Energy Phys., 1995. P. 126; J. Phys. A. 1994. V. 27. P. 2053.

[12] Ю. С. Вернов, М. Н. Мнацаканова. ТМФ. 1997. Т. 113. С. 355.

[13] Ю. С. Вернов, М. Н. Мнацаканова. ТМФ. 2000. Т. 125. С. 272.

[14] Ю. С. Вернов, М. Н. Мнацаканова. ТМФ. 2001. Т. 129. С. 219.

[15] F. Rellich. Nachr. Akad. Wiss. Gött. Math-Phys. Kl., Math. -Phys. -Chem. Abt. 1946. V. 1946. P. 107; J. Dixmier. Compos. Math. 1958. V. 13. P. 263. 